\title{
Unlocking CO Depletion in Protoplanetary Disks. II. Primordial C/H Predictions inside the CO Snowline
}

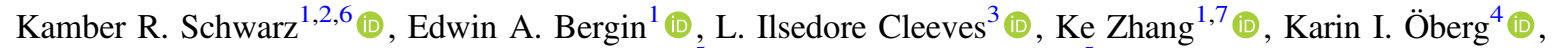 \\ Geoffrey A. Blake ${ }^{5}$ (D), and Dana E. Anderson ${ }^{5}$ (i) \\ ${ }^{1}$ Department of Astronomy, University of Michigan, 1085 South University Ave., Ann Arbor, MI 48109, USA; kschwarz@1pl.arizona.edu \\ ${ }^{2}$ Lunar and Planetary Laboratory, University of Arizona, 1629 E. University Blvd., Tucson, AZ 85721, USA \\ ${ }^{3}$ Department of Astronomy, University of Virginia, 530 McCormick Rd., Charlottesville, VA 22904, USA \\ ${ }^{4}$ Harvard-Smithsonian Center for Astrophysics, 60 Garden St., Cambridge, MA 02138, USA \\ ${ }^{5}$ Division of Geological \& Planetary Sciences, MC 150-21, California Institute of Technology, 1200 E California Blvd., Pasadena, CA 91125, USA \\ Received 2019 February 4; revised 2019 April 7; accepted 2019 April 23; published 2019 June 4
}

\begin{abstract}
$\mathrm{CO}$ is thought to be the main reservoir of volatile carbon in protoplanetary disks, and thus the primary initial source of carbon in the atmospheres of forming giant planets. However, recent observations of protoplanetary disks point toward low volatile carbon abundances in many systems, including at radii interior to the CO snowline. One potential explanation is that gas phase carbon is chemically reprocessed into less volatile species, which are frozen on dust grain surfaces as ice. This mechanism has the potential to change the primordial $\mathrm{C} / \mathrm{H}$ ratio in the gas. However, current observations primarily probe the upper layers of the disk. It is not clear if the low volatile carbon abundances extend to the midplane, where planets form. We have run a grid of 198 chemical models, exploring how the chemical reprocessing of $\mathrm{CO}$ depends on disk mass, dust grain size distribution, temperature, cosmic-ray and X-ray ionization rate, and initial water abundance. Building on our previous work focusing on the warm molecular layer, here we analyze the results for our grid of models in the disk midplane at 12 au. We find that either an ISM level cosmic-ray ionization rate or the presence of UV photons due to a low dust surface density are needed to chemically reduce the midplane $\mathrm{CO}$ gas abundance by at least an order of magnitude within $1 \mathrm{Myr}$. In the majority of our models $\mathrm{CO}$ does not undergo substantial reprocessing by in situ chemistry and there is little change in the gas phase $\mathrm{C} / \mathrm{H}$ and $\mathrm{C} / \mathrm{O}$ ratios over the lifetime of the typical disk. However, in the small subset of disks where the disk midplane is subject to a source of ionization or photolysis, the gas phase $\mathrm{C} / \mathrm{O}$ ratio increases by up to nearly 9 orders of magnitude due to conversion of $\mathrm{CO}$ into volatile hydrocarbons.
\end{abstract}

Key words: astrochemistry - circumstellar matter - ISM: abundances - ISM: molecules - protoplanetary disks

Supporting material: machine-readable table

\section{Introduction}

Planets are formed out of the gas and dust in the protoplanetary disks around young stars. The composition of these planets is thus primarily set by the composition of the parent disk. A planet formed via gravitational instability should have an atmospheric composition similar to that of the bulk composition of the disk, i.e., that of the host star. For planets formed via core accretion, the connection between disk and planet compositions is more complex.

A factor in determining a planet's composition in the core accretion scenario is its formation location relative to the snowlines of major volatiles (Öberg et al. 2011). A snowline is the location in a disk where a species such as $\mathrm{CO}, \mathrm{CO}_{2}$, or $\mathrm{H}_{2} \mathrm{O}$ transitions from being frozen out as ice to being in the gas phase. These condensation fronts result in sharp transitions in the $\mathrm{C} / \mathrm{O}$ ratio in both the gas and the solids. The $\mathrm{C} / \mathrm{O}$ ratio in the gas and solids can be further modified by disk dynamics. As dusty particles drift inward they can remove volatile ices from the outer disk (Ciesla \& Cuzzi 2006). At small radii these ices will sublimate, enriching the inner disk gas in volatiles (Estrada et al. 2016; Öberg \& Bergin 2016). Disk evolution can also change the snowline locations due to either radial drift of dust

\footnotetext{
6 Sagan Fellow.

7 Hubble Fellow.
}

(Piso et al. 2015) or an evolving temperature structure (Eistrup et al. 2018). Additionally, chemical reprocessing can change the relative abundance of volatiles in the gas and ice (Eistrup et al. 2016; Yu et al. 2016).

Models combining various aspects of this evolution: disk chemistry, planet migration, and atmospheric chemistry, point toward the use of the $\mathrm{C} / \mathrm{O}$ ratio in a planet's atmosphere as a way to trace the formation history of the planet when combined with additional information such as the $\mathrm{C} / \mathrm{H}$ or $\mathrm{C} / \mathrm{N}$ ratio (Booth et al. 2017; Cridland et al. 2017). Several studies have now derived $\mathrm{C} / \mathrm{O}$ ratios for giant exoplanets, either directly from the detection of spectra in the atmosphere or through a combination of observations and models (Kreidberg et al. 2015; Espinoza et al. 2017; Lavie et al. 2017). As observations and models continue to improve we are fast approaching an era of connecting protoplanetary disk and planet compositions.

Observations of a growing number of protoplanetary disks reveal low $\mathrm{CO}$ abundances relative to that expected from the dust mass (Ansdell et al. 2016; Long et al. 2017). Models of $\mathrm{CO}$ in these disks show that this discrepancy cannot be fully explained by $\mathrm{CO}$ freeze-out, nor is it due to a failure to properly correct for isotopologue selective self-shielding (Miotello et al. 2014; Williams \& Best 2014). CO is not the only volatile molecule with low observed abundances. Observations of $\mathrm{H}_{2} \mathrm{O}$ vapor and atomic carbon also reveal these species to be underabundant (Kama et al. 2016; Du et al. 2017). This "missing 
volatiles problem" has several potential solutions including gas disk dispersal, gaseous interactions with the evolving dust population, and chemical reprocessing (Reboussin et al. 2015; Bai 2016; Krijt \& Ciesla 2016; Xu et al. 2017). However, as disk gas masses are usually derived from either $\mathrm{CO}$ or dust observations, it is often difficult to distinguish between the different scenarios.

One way to differentiate between disk dispersal and mechanisms that affect only the volatiles is through observations of the $\mathrm{H}_{2}$ isotopologue $\mathrm{HD}$, which is more closely related to the total gas mass than either $\mathrm{CO}$ or dust and primarily emits from warm $(20-50 \mathrm{~K})$ gas within the inner few tens of astronomical units (Zhang et al. 2017). HD has been successfully detected in three disks to date (Bergin et al. 2013; McClure et al. 2016), and reveals CO to be underabundant by roughly two orders of magnitude in TW Hya (Favre et al. 2013; Schwarz et al. 2016). For DM Tau and GM Aur, the other disks with HD detections, CO appears underabundant by an order of magnitude, though uncertainties related to the disk thermal structure remain (McClure et al. 2016). These lines of evidence point toward processes beyond gas disk dispersal contributing to low CO-to-dust ratios.

Millimeter observations of $\mathrm{CO}$ almost exclusively probe regions in the disk above the midplane and outside the $\mathrm{CO}$ snowline, in the warm molecular layer. In only one system have optically thin $\mathrm{CO}$ isotopologues been observed inside the $\mathrm{CO}$ snowline: TW Hya. Using observations of optically thin ${ }^{13} \mathrm{C}^{18} \mathrm{O}$ emission interior to the midplane $\mathrm{CO}$ snowline in $\mathrm{TW}$ Hya, Zhang et al. (2017) find an average ${ }^{13} \mathrm{C}^{18} \mathrm{O}$ abundance of $1.7 \times 10^{-10}$ relative to $\mathrm{H}_{2}$ for gas warmer than $20 \mathrm{~K}$ in the radial range 5-20 au. This corresponds to an average $\mathrm{CO}$ abundance in the same region of $6.5 \times 10^{-6}$. As $\mathrm{CO}$ is expected to be the dominant gas phase carbon species between the $\mathrm{CO}$ and $\mathrm{CO}_{2}$ snowlines, this suggests that the midplane $\mathrm{C} / \mathrm{H}$ gas ratio is well below expectations in this one system. Previous studies demonstrate substantial chemical reprocessing of $\mathrm{CO}$ in the midplane is possible, particularly in the presence of cosmic rays (Eistrup et al. 2016; Yu et al. 2016). In this work we explore the viability of chemical reprocessing as a way to remove volatile species from the gas for models spanning a large range of physical conditions, with a focus on different disk masses and large grain fractions.

In Schwarz et al. (2018), hereafter Paper I, we analyzed the results of our grid of chemical models for the warm molecular layer. In this paper we focus on the midplane $\mathrm{CO}$ gas abundance at $12 \mathrm{au}$, which is between the midplane $\mathrm{CO}$ and $\mathrm{CO}_{2}$ snowlines for all models, as well as within the expected formation region for giant planets (Chabrier et al. 2014). Section 2 briefly summaries our model framework and parameter space. The results are described in Section 3. In Section 4 we compare our results to previous studies and solar system bodies, as well as discuss the implications for planet formation. Finally, our findings are summarized in Section 5.

\section{Model}

We use the model described in detail in Paper I, summarized below. We explore a range of parameters: disk mass, dust grain size distribution, temperature, X-ray and cosmic-ray ionization, and initial water abundance (Table 1). Our model setup is a two-dimensional, azimuthally symmetric disk. The density and temperature structure, as well as the dust opacity, are generated using the radiative transfer code TORUS (Harries 2000). We
Table 1

Physical Model Properties

\begin{tabular}{ll}
\hline \hline Parameter & Values \\
\hline$M_{\text {disk }}\left(M_{\odot}\right)$ & $0.1,0.03,0.003$ \\
$L_{\mathrm{XR}}\left(\mathrm{erg} \mathrm{s}^{-1}\right)$ & $1 \mathrm{E} 30,1 \mathrm{E} 31$ \\
$\zeta_{\mathrm{CR}}\left(\mathrm{s}^{-1}\right)$ & $1.6 \mathrm{E}-19,2 \mathrm{E}-17$ \\
$f_{l}$ & $0.0,0.1,0.2,0.3,0.4,0.5$, \\
$R_{\text {in }}(\mathrm{au})$ & $0.6,0.7,0.8,0.9,0.99$ \\
$R_{\text {out }}(\mathrm{au})$ & 0.1 \\
\hline
\end{tabular}

Table 2

Initial Abundances Relative to $\mathrm{H}_{2}$

\begin{tabular}{lccc}
\hline \hline Species & Abundance & Species & Abundance \\
\hline $\mathrm{H}_{2}$ & $1.00 \mathrm{E}-00$ & $\mathrm{H}_{3}^{+}$ & $2.00 \mathrm{E}-08$ \\
$\mathrm{He}$ & $2.80 \mathrm{E}-01$ & $\mathrm{HCO}^{+}$ & $1.80 \mathrm{E}-08$ \\
$\mathrm{CO}$ & $2.00 \mathrm{E}-04$ & $\mathrm{C}_{2} \mathrm{H}$ & $1.60 \mathrm{E}-08$ \\
$\mathrm{H}_{2} \mathrm{O}(\mathrm{gr})$ & $1.20 \mathrm{E}-04$ & $\mathrm{H}_{2} \mathrm{CO}$ & $1.60 \mathrm{E}-08$ \\
$\mathrm{~N}$ & $4.50 \mathrm{E}-05$ & $\mathrm{SO}$ & $1.00 \mathrm{E}-08$ \\
$\mathrm{CO}_{2}(\mathrm{gr})$ & $4.00 \mathrm{E}-05$ & $\mathrm{CS}$ & $8.00 \mathrm{E}-09$ \\
$\mathrm{~N}_{2}$ & $2.00 \mathrm{E}-06$ & $\mathrm{C}^{+}$ & $2.00 \mathrm{E}-09$ \\
$\mathrm{C}$ & $1.40 \mathrm{E}-06$ & $\mathrm{Si}^{+}$ & $2.00 \mathrm{E}-11$ \\
$\mathrm{NH}_{3}$ & $1.60 \mathrm{E}-07$ & $\mathrm{~S}^{+}$ & $2.00 \mathrm{E}-11$ \\
$\mathrm{CN}$ & $1.20 \mathrm{E}-07$ & $\mathrm{Mg}^{+}$ & $2.00 \mathrm{E}-11$ \\
$\mathrm{HCN}$ & $4.00 \mathrm{E}-08$ & $\mathrm{Fe}^{+}$ & $2.00 \mathrm{E}-11$ \\
\hline
\end{tabular}

consider disks with an inner radius of 0.1 au and an outer radius of $200 \mathrm{au}$, and masses of $0.1,0.03$, and $0.003 M_{\odot}$. Our choice of outer disk radius is larger than the radius of a "typical" protoplanetary disk. However, the midplane chemistry in our models is not sensitive to the outer radius of the disk (see Appendix A). This is due to several factors: the only source of radiation in our models is the central star, we do not physically evolve the disk, and the chemical evolution at each radius is calculated independently

Each disk has two dust populations. The first treats small grains $\left(r_{d}=0.005-1 \mu \mathrm{m}\right)$, which are well mixed with the gas. Large grains $\left(r_{d}=0.005-1000 \mu \mathrm{m}\right)$ are more settled than the small grains and gas as described in Paper I. Both the small and large grain populations have an MRN size distribution (Mathis et al. 1977). The fractional dust mass in large grains varies from 0 (all dust in small grains) to 0.99 in 11 steps.

All of our disk models are irradiated by a central T Tauri star with a mass of $0.8 M_{\odot}$ and an effective temperature of $4300 \mathrm{~K}$. The radiative transport of the UV and X-ray photons through the disk are computed using the methods described by Bethell \& Bergin (2011a, 2011b). Our chemical evolution model is based on that of Cleeves et al. (2014b) and the chemical networks of Smith et al. (2004), Fogel et al. (2011), and McElroy et al. (2013). The network includes an extensive number of gas phase reactions, including ionization by cosmic rays and $\mathrm{X}$-rays, as well as a limited number of grain surface reactions focusing on the grain surface formation of $\mathrm{H}_{2} \mathrm{O}$ and $\mathrm{CO}_{2}$ as well as the hydrogenation of volatile carbon and nitrogen. The initial abundances are listed in Table 2 and are based on the model molecular cloud abundances of Aikawa \& Herbst (1999). The exceptions are the initial $\mathrm{H}_{2} \mathrm{O}$ ice and $\mathrm{CO}_{2}$ ice abundances, which have been adjusted to match the carbon and oxygen partitioning assumed by Öberg et al. (2011) in 


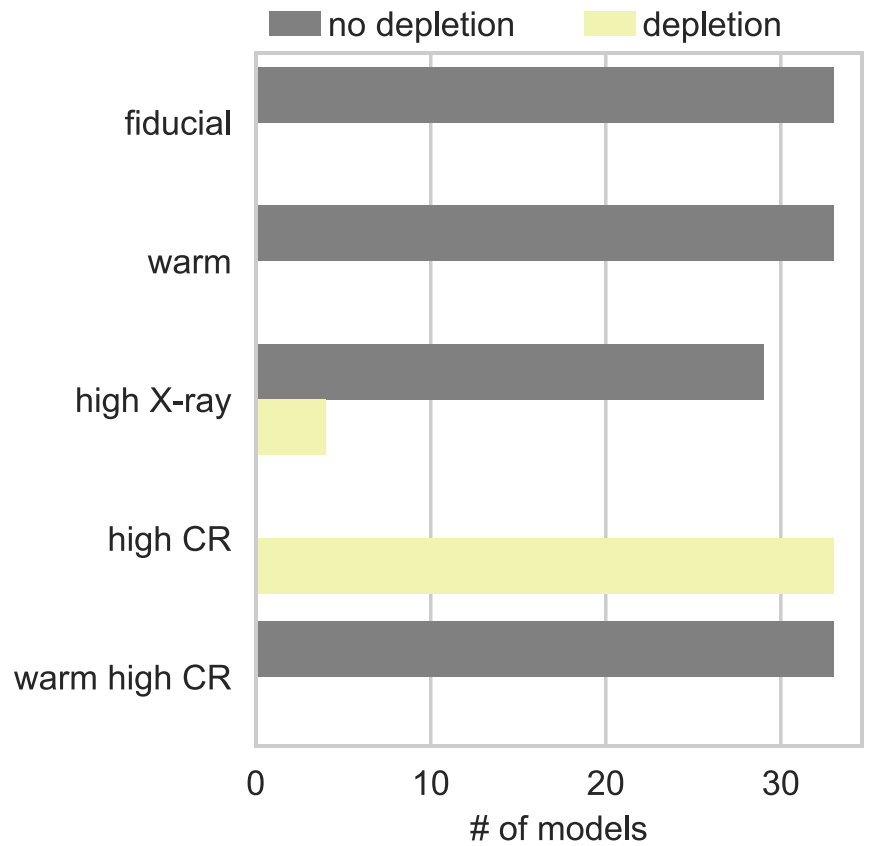

Figure 1. Breakdown of the number of models that are depleted (X) $\mathrm{CO}$ gas $\left.)<10^{-4}\right)$ and not depleted $\left(\mathrm{X}(\mathrm{CO}\right.$ gas $\left.)>10^{-4}\right)$ at $1 \mathrm{Myr}$. Twelve of the high $\mathrm{CR}$ models are extremely depleted $\left(\mathrm{X}(\mathrm{CO}\right.$ gas $\left.)<10^{-5}\right)$.

order to facilitate comparison with their $\mathrm{C} / \mathrm{O}$ values. We evolve the chemistry for $6 \mathrm{Myr}$ while the disk physical conditions remain static

Our fiducial models assume a cosmic-ray ionization rate of $1.6 \times 10^{-19} \mathrm{~s}^{-1}$ and an X-ray luminosity of $10^{30} \mathrm{erg} \mathrm{s}^{-1}$ for three disk masses and 11 different large grain fractions. This lower cosmic-ray ionization rate replicates the modulation of cosmic rays by winds (Cleeves et al. 2014a). We also consider high $\mathrm{X}$-ray models $\left(10^{31} \mathrm{erg} \mathrm{s}^{-1}\right)$, high cosmic-ray rate models $\left(2 \times 10^{-17} \mathrm{~s}^{-1}\right)$ equivalent to the flux in the diffuse ISM, warm models where the gas and dust temperature has been uniformly enhanced by $20 \mathrm{~K}$, even in the midplane, and warm high cosmic-ray rate models for a total of 165 different models. Additionally, we remove the initial water abundance for the fiducial, high X-ray, and high cosmic-ray models with a disk mass of $0.03 M_{\odot}$, for a total of 198 unique models.

\section{Results}

We choose to focus on a single location in the disk, the midplane at $12 \mathrm{au}$, in order to make the analysis of a large number of models more manageable. We assume that $12 \mathrm{au}$ is representative of the disk midplane between the $\mathrm{CO}$ and $\mathrm{CO}_{2}$ snowlines. The validity of this assumption is discussed at the end of this section.

Figure 1 summaries our findings for models with a standard ISM abundance. A model is considered depleted if the gas phase $\mathrm{CO}$ abundance relative to $\mathrm{H}_{2}$ is less than half the initial abundance, that is $\mathrm{X}(\mathrm{CO})<10^{-4}$. A model is considered extremely depleted when the gas phase $\mathrm{CO}$ abundance drops below $10^{-5}$, or 20 times lower than the initial abundance. At 12 au after $0.1 \mathrm{Myr}, 1 \%$ of models are depleted by a factor of 2 to 20 in gas phase $\mathrm{CO}$, increasing to $15 \%$ after $1 \mathrm{Myr}, 11 \%$ after $3 \mathrm{Myr}$, and $10 \%$ after $6 \mathrm{Myr}$. Additionally, $7 \%$ of models are depleted by more than a factor of 20 after $1 \mathrm{Myr}$, increasing to $30 \%$ after $3 \mathrm{Myr}$ and $39 \%$ after $6 \mathrm{Myr}$. The majority of the models are not depleted in midplane $\mathrm{CO}$ after $1 \mathrm{Myr}$, with $\mathrm{X}(\mathrm{CO})>10^{-4}$. The models that are able to reduce the $\mathrm{CO}$ gas abundance by more than two orders of magnitude within $1 \mathrm{Myr}$ are the $0.03 M_{\odot}$ disks with a high cosmic-ray ionization rate, while the $0.1 M_{\odot}$ high cosmic-ray models are depleted by an order of magnitude after $1 \mathrm{Myr}$. The midplane $\mathrm{CO}$ abundance after $1 \mathrm{Myr}$ in each model is shown in Figure 2. Additionally, the abundances of the top five carbon bearing species in each model are given in Appendix B.

In the high cosmic-ray rate models, the carbon has been chemically reprocessed into $\mathrm{CH}_{3} \mathrm{OH}$ ice. Cosmic rays are able to create $\mathrm{H}_{3}^{+}$, which reacts with gas phase $\mathrm{CO}$ to form $\mathrm{HCO}^{+}$. This almost immediately recombines with an electron, placing the carbon once again in CO. However, this process is also a way to free hydrogen atoms from $\mathrm{H}_{2}$. Some of these hydrogen atoms freeze out onto grains where they are able to hydrogenate $\mathrm{CO}$ ice before it can be thermally desorbed back into the gas. Successive hydrogenation on the grain surface ultimately culminates in the formation of $\mathrm{CH}_{3} \mathrm{OH}$ ice. Because the process of converting $\mathrm{CO}$ gas into $\mathrm{CH}_{3} \mathrm{OH}$ ice does not require any additional oxygen beyond that in $\mathrm{CO}$, it is still effective in the models where the initial water is removed. Thus, the $\mathrm{CO}$ abundances in the fiducial, high X-ray, and high cosmic-ray models without an initial reservoir or water ice reflect the abundances in the corresponding models with a fiducial water abundance. $\mathrm{CH}_{3} \mathrm{OH}$ ice is an end state product in our chemical network. Additional processing on the grain surface is possible, leading to the formation of, e.g., hydrocarbon ices on timescales of several megayears (Bosman et al. 2018). As such the $\mathrm{CH}_{3} \mathrm{OH}$ ice abundances in our models should be considered upper limits while the overall complexity of the chemistry should be considered a lower limit since $\mathrm{CH}_{3} \mathrm{OH}$ can be a precursor to more complex species.

For the $0.003 M_{\odot}$ disk, the fiducial, high X-ray, and high cosmic-ray models with $99 \%$ of their mass in large grains are depleted in $\mathrm{CO}$ by a factor of two after 1 Myr. In these models carbon is primarily in $\mathrm{CO}_{2}$ ice. In these low density, highly settled disks, UV photons are able to reach the midplane. These photons dissociate $\mathrm{H}_{2} \mathrm{O}$ ice, creating $\mathrm{OH}$ gas. The $\mathrm{OH}$ then freezes back onto the grain where it reacts with $\mathrm{CO}$ ice to form $\mathrm{CO}_{2}$ ice. As in higher mass disks, the high cosmic-ray models also allow for additional chemical reprocessing of $\mathrm{CO}$. Likewise, the ionization provided by the X-rays in the high $\mathrm{X}$-ray models also leads to approximately half of the $\mathrm{CO}$ being reprocessed. However, the higher temperatures in these models reduce the amount of $\mathrm{CO}$ on the grain surface, resulting in longer timescales for the reprocessing into $\mathrm{CO}_{2}$ and $\mathrm{CH}_{3} \mathrm{OH}$ ice. A similar behavior is seen in all of the models with both artificially increased temperature and high cosmic-ray rates.

\subsection{Radial Abundance Variations}

To test our assumption that the midplane abundances at $12 \mathrm{au}$ are representative of the disk between the $\mathrm{CO}$ and $\mathrm{CO}_{2}$ snowlines we analyze the variation in the radial abundance structure of carbon bearing species in the midplane for four representative disks: the fiducial $0.03 M_{\odot}$ disk model with $50 \%$ large grains, which does not show evidence for substantial $\mathrm{CO}$ reprocessing, as well as the high cosmic-ray rate, 50\% large grain models for all three disk masses. The midplane abundance of the major carbon bearing species in these models are shown after $1 \mathrm{Myr}$ in Figure 3 and after $6 \mathrm{Myr}$ in Figure 4. 


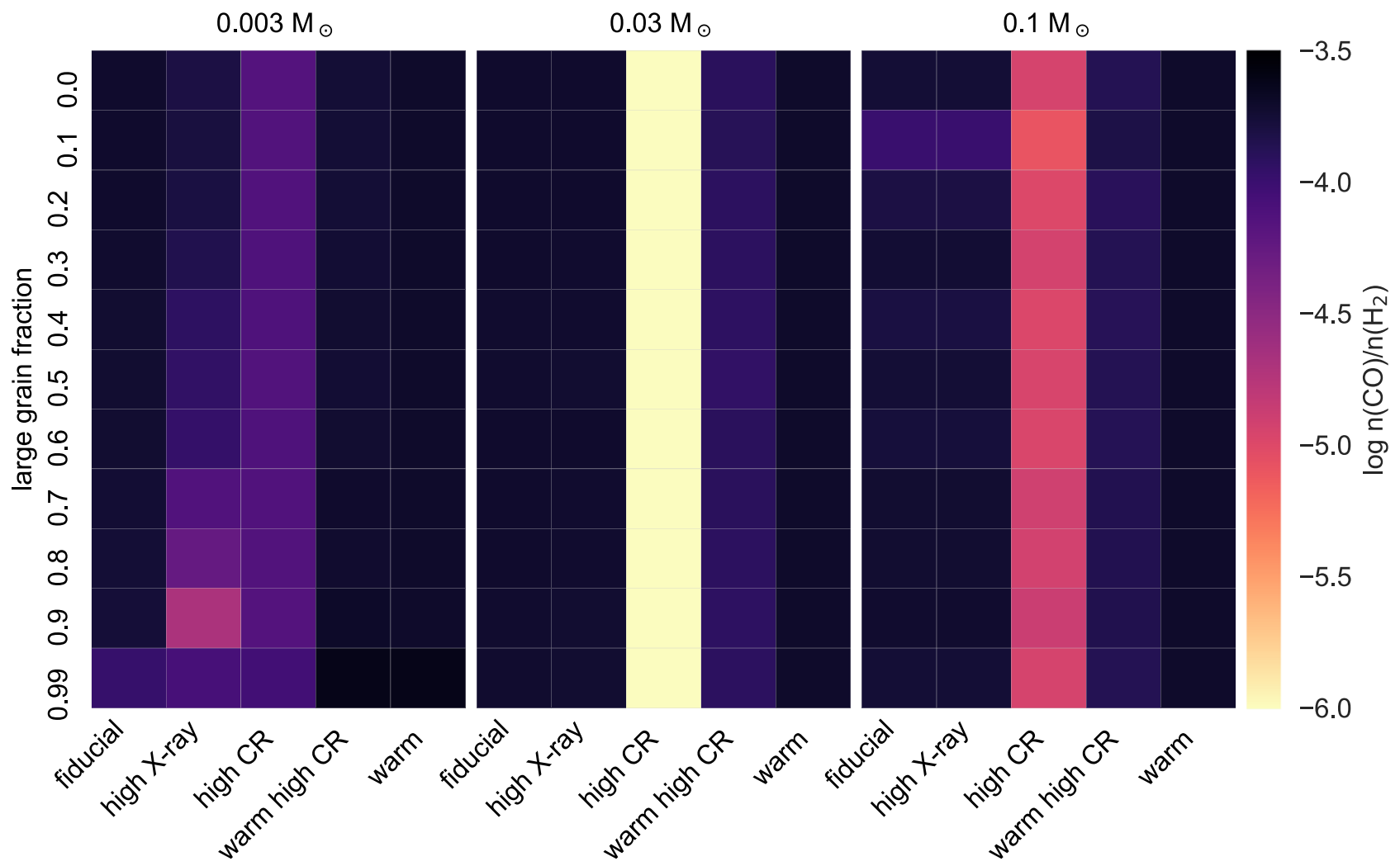

Figure 2. $\log \mathrm{CO}$ gas abundance relative to $\mathrm{H}_{2}$ in the midplane at 12 au for each model after 1 Myr.

In each of these models $12 \mathrm{au}$ is located between the $\mathrm{CO}$ and $\mathrm{CO}_{2}$ snowlines.

The timescales for $\mathrm{CO}$ reprocessing are shorter in the outer disk, such that after $1 \mathrm{Myr}$ gas phase CO depletion has occurred over a limited radial range. 12 au is within this range for the 0.1 and $0.03 M_{\odot}$ disks with a high cosmic-ray rate, while in the lower density $0.003 M_{\odot}$ model significant $\mathrm{CO}$ reprocessing has occurred only at larger radii. After $6 \mathrm{Myr}$, the $\mathrm{CO}$ abundance between the $\mathrm{CO}_{2}$ and $\mathrm{CO}$ snowlines is more uniform, though the $\mathrm{CO}$ gas abundance does increase at the snowlines of carbon bearing ices such as $\mathrm{CH}_{3} \mathrm{OH}$ and $\mathrm{HCN}$. In each model the change in $\mathrm{CO}$ abundance from the $\mathrm{CO}$ snowline to just outside the $\mathrm{CO}_{2}$ snowline is minor enough to not affect whether a disk is classified as depleted in $\mathrm{CO}$ according to our criteria. Therefore we conclude that the abundances at 12 au are representative of the disk between these two major snowlines, with the caveat that depletion timescales will be longer at smaller radii.

\section{Discussion}

\subsection{Chemistry as a Depletion Mechanism}

In our models, there are two sets of conditions that lead to substantial chemical reprocessing of gas phase $\mathrm{CO}$ in the inner disk midplane. The first is if the disk has a low enough mass surface density of small grains or overall gas + dust physical density for UV radiation to reach the midplane, reprocessing the carbon primarily into $\mathrm{CO}_{2}$ ice. The second, and more widespread, condition is to expose the disk to a high ionization rate, comparable to the cosmic-ray ionization rate seen in the ISM, in which case much of the carbon is placed into $\mathrm{CH}_{3} \mathrm{OH}$ ice.
Our results, namely that ionization is an important mechanism and that carbon goes into $\mathrm{CO}_{2}$ and $\mathrm{CH}_{3} \mathrm{OH}$ ices, agree with previous studies of chemical reprocessing (Eistrup et al. 2016; Yu et al. 2016). These studies also included an ISM level cosmic-ray ionization rate. However, it is possible that Class II disks see a lower cosmic-ray rate due to modulation by a stellar wind (Cleeves et al. 2014b). This modulation of cosmic rays is seen in our own solar system, while observations of ionized molecules in TW Hya also indicate a low cosmic-ray ionization rate (Cleeves et al. 2015). An alternative ionization source is stellar X-rays, which can also result in chemical reprocessing of $\mathrm{CO}$ for a range of physical conditions (Dodson-Robinson et al. 2018) However, the ionization structure in most systems remains largely unconstrained by observations. Analysis of the $\mathrm{N}_{2} \mathrm{H}^{+}$and $\mathrm{HCO}^{+}$emission in these systems, similar to the work by Cleeves et al. (2015) in TW Hya, will provide crucial observational constraints on the typical ionization level in protoplanetary disks.

It is possible that depletion primarily occurs in younger Class 0 and Class I systems, which may have a higher cosmic-ray ionization rate (Padovani et al. 2016). In fact, there is emerging observational evidence of cosmic-ray acceleration in the bow shock region of low-mass protostars (Tychoniec et al. 2018). Observations of the envelopes of some Class 0 protostars reveal reduced $\mathrm{CO}$ gas abundances, suggesting $\mathrm{CO}$ depletion may begin early (Anderl et al. 2016). Conversely, there is no evidence of substantial $\mathrm{CO}$ depletion in the embedded Class I disk L1527 (van't Hoff et al. 2018). Until we understand more about the ionization structure in protoplanetary disks as a population it will be difficult to determine the extent to which chemical reprocessing contributes to the removal of volatiles from the gas. 


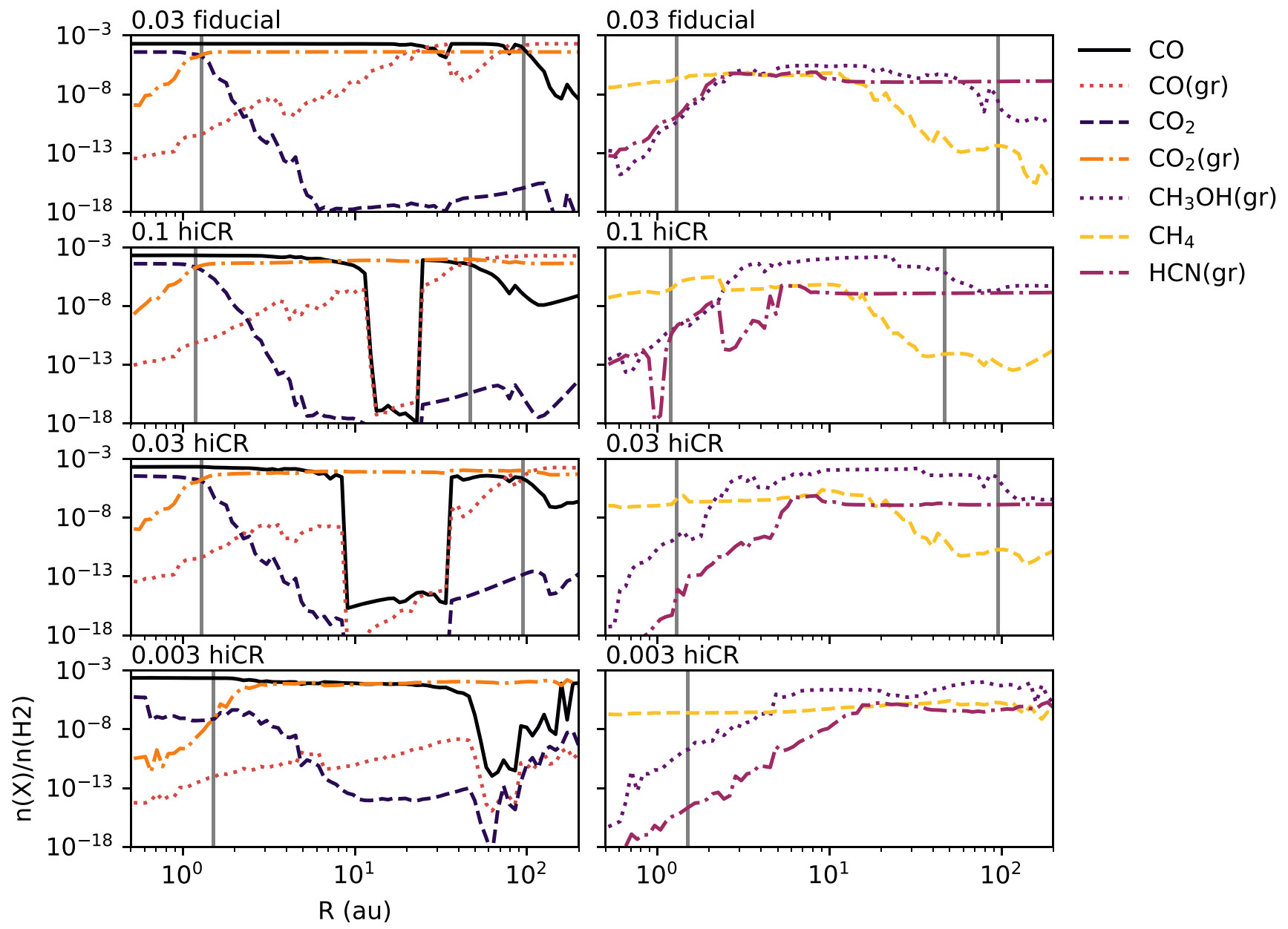

Figure 3. Midplane abundances of carbon bearing species as a function of radius for four representative models after 1 Myr. All models have $50 \%$ of their dust mass in large grains. The $\mathrm{CO}_{2}$ and $\mathrm{CO}$ snowlines, defined as the largest radius where the gas and ice abundance of the species are equal, are shown by vertical gray lines. For the $0.003 M_{\odot}$ disk the entirety of the disk is inside the CO snowline.

Dynamical processes could also contribute to CO depletion. Vertical mixing brings gas phase species from the upper layers of the disk to the cold midplane, where they freeze out onto dust grains, thus depleting the upper layers of the disk in what is sometimes referred to as the vertical "cold finger" effect (Meijerink et al. 2009). This freeze out of gas can result in a factor of 50 depletion in the upper layers, while enriching the midplane ices in volatiles (Xu et al. 2017). As these icy grains drift inward they enrich the gas inside a given species' snowline (Krijt et al. 2018). This will counter the depletion due to chemical reprocessing in the inner disk so long as grains continue to drift inward. Indeed, Booth et al. (2017) find that the inward drift of icy pebbles can lead to the formation of giant planets with both super solar $\mathrm{C} / \mathrm{H}$ and super solar $\mathrm{C} / \mathrm{O}$ exterior to the $\mathrm{H}_{2} \mathrm{O}$ snowline. If the dust grains are prevented from drifting inward, e.g., due to a pressure bump, this volatile enrichment will not be observed. Alternatively, if, as in our models, $\mathrm{CO}$ ice is converted into species with higher binding energies there will not be an enrichment of gas phase carbon as grains pass inside the $\mathrm{CO}$ snowline. However, so long as ice coated grains continue to drift inward the ices will eventually sublimate, resulting in greater enrichment at smaller radii.

\subsection{Additional Factors}

Our models focus on how chemistry in disks with a range of masses and grain size distributions is effected by limiting cases for the cosmic-ray ionization rate and X-ray luminosity of $\mathrm{T}$-Tauri stars. We also assume that the chemistry is evolving in a physically static system. In reality, over the course of the $6 \mathrm{Myr}$ we consider the disk, and the central star, will be evolving, leading to changes in the dust size distribution, dust spatial distribution, and stellar luminosity. The luminosity of the central star, particularly in the FUV, can potentially impact the disk chemistry in two ways. First, FUV radiation heats the gas disk through the photoelectric effect (Weingartner \& Draine 2001). The star cools with time and its UV luminosity decreases, resulting in a cooler disk. As the disk cools the snowlines of major volatiles move inward, with the largest change seen for snowlines that start at large radii (Yu et al. 2016; Eistrup et al. 2018). Our models show that warmer disk temperatures, such as those expected for younger disks, hinder chemical reprocessing (Figure 2), a result also found by several previous studies (Reboussin et al. 2015; Bosman et al. 2018). However, as the star evolves, the decrease in FUV luminosity also means the flux of ionizing photons in the disk decreases. This will make it more difficult for $\mathrm{CO}$ to be reprocessed into 


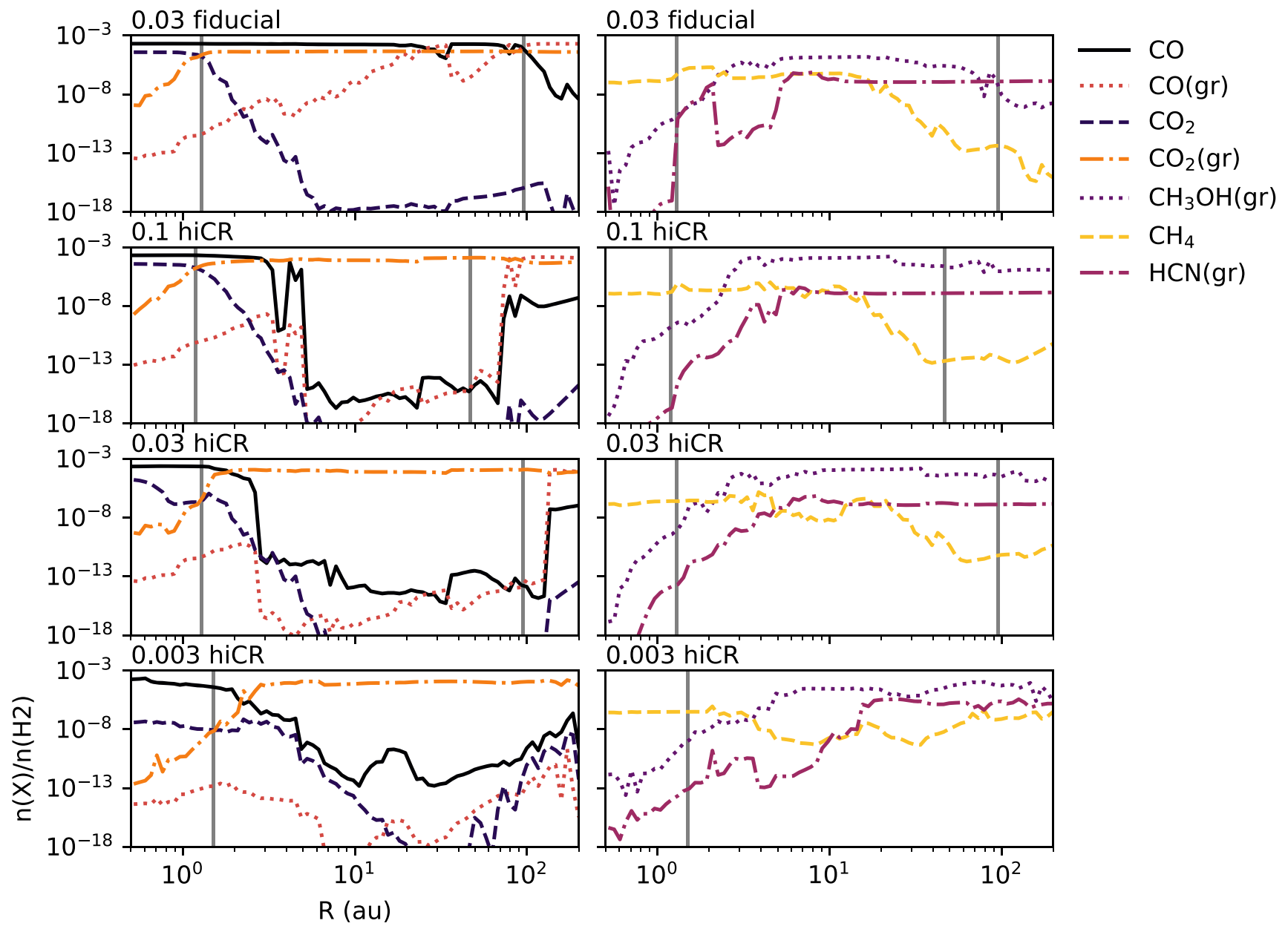

Figure 4. Midplane abundances of carbon bearing species as a function of radius for four representative models after 6 Myr. All models have $50 \%$ of their dust mass in large grains. The $\mathrm{CO}_{2}$ and $\mathrm{CO}$ snowlines, defined as the largest radius where the gas and ice abundance of the species are equal, are shown by vertical gray lines. For the $0.003 M_{\odot}$ disk the entirety of the disk is inside the CO snowline.

$\mathrm{CO}_{2}$ ice, as this process relies on the photodissociation of $\mathrm{H}_{2} \mathrm{O}$ ice. So while a cooling disk will promote the reprocessing of $\mathrm{CO}$, a decreasing UV flux will hinder it. Additional work exploring how these two effects work in concert is needed to determine which one is dominant.

There is currently some debate in regards to whether disks inherit their initial abundances from the ISM or if the chemistry is at least partially reset by heating during collapse. However, after several million years of chemical evolution, models with atomic initial abundances have a very similar composition to models with molecular initial abundances (Molyarova et al. 2017; Eistrup et al. 2018). More generally, a reduction in the initial amount of $\mathrm{H}_{2} \mathrm{O}$ ice will slow down the conversion of $\mathrm{CO}$ to $\mathrm{CO}_{2}$ ice. To form $\mathrm{CO}_{2}, \mathrm{CO}$ reacts with $\mathrm{OH}$ on the grain surface and $\mathrm{OH}$ is formed from dissociated $\mathrm{H}_{2} \mathrm{O}$.

\subsection{Consequences for Planet Composition}

\subsection{1. $\mathrm{C} / \mathrm{H}$}

In this section we analyze the total gas phase $\mathrm{C} / \mathrm{H}$ ratio in the midplane at 12 au to assess how much bulk gas phase carbon planets can accrete in their atmospheres. Figure 5 shows the $\mathrm{C} / \mathrm{H}$ ratio in the gas considering only the snowline locations for the major volatiles, assuming $62.5 \%$ of the total carbon content is in $\mathrm{CO}, 12.5 \%$ is in $\mathrm{CO}_{2}$, and the remaining $25 \%$ is in refractory material such as carbon grains. Between the $\mathrm{CO}$ and $\mathrm{CO}_{2}$ snowlines all of the gas phase carbon is in $\mathrm{CO}$, so the gas phase $\mathrm{C} / \mathrm{H}$ ratio is 0.625 relative to stellar. Inside the $\mathrm{CO}_{2}$ snowline $\mathrm{CO}_{2}$ returns to the gas and the gas $\mathrm{C} / \mathrm{H}$ ratio rises to 0.75 .

Also shown in Figure 5 is the distribution of gas phase $\mathrm{C} / \mathrm{H}$ values in the midplane at 12 au for our models. In this framework, the largest possible $\mathrm{C} / \mathrm{H}$ value in our models, relative to stellar, is 0.75 . Between the $\mathrm{CO}$ and $\mathrm{CO}_{2}$ snowlines, the majority of our models have a $\mathrm{C} / \mathrm{H}$ ratio close to what is expected based on snowline locations after $1 \mathrm{Myr}$ of chemical evolution. For these models the $\mathrm{CO}$ has undergone very little reprocessing and it remains in the gas at close to the initial abundance. However, there is a subset of models where the gas phase $\mathrm{C} / \mathrm{H}$ value is substantially reduced. These are the models with an enhanced cosmic-ray ionization rate as well as the $0.003 M_{\odot}$ disk high X-ray models, i.e., those with significant $\mathrm{CO}$ reprocessing (see Figure 2).

After $6 \mathrm{Myr}$, chemical processes result in substantially lower gas phase $\mathrm{C} / \mathrm{H}$ for most models. The models with some reduction in gas phase carbon after $1 \mathrm{Myr}$ retain very little gas phase carbon after $6 \mathrm{Myr}$. However, the 0.03 and $0.1 M_{\odot}$. models with a fiducial cosmic-ray ionization rate $(1.6 \times$ $10^{-19} \mathrm{~s}^{-1}$ ) retain much of their initial $\mathrm{CO}$ gas even after $6 \mathrm{Myr}$ of evolution. In these models the gas $\mathrm{C} / \mathrm{H}$ ratio has changed by less than a factor of two after $6 \mathrm{Myr}$ of chemical 

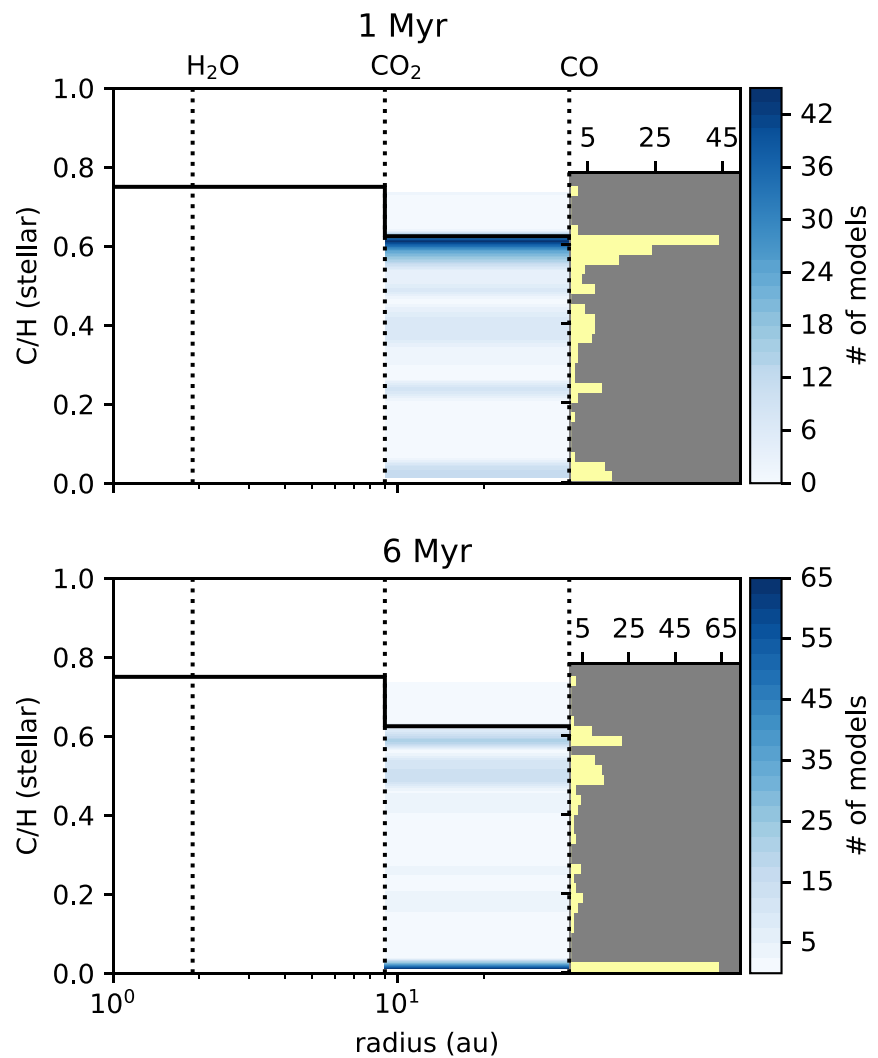

Figure 5. $\mathrm{C} / \mathrm{H}$ ratio in the gas based on the location of major volatile snowlines (line) and the distribution of $\mathrm{C} / \mathrm{H}$ values inside the $\mathrm{CO}$ snowline for our models (shading and histogram) after $1 \mathrm{Myr}$ (top) and $6 \mathrm{Myr}$ (bottom). Radial changes in the $\mathrm{C} / \mathrm{H}$ value are based on the carbon partitioning assumptions of Öberg et al. (2011).

evolution. In summary, models with greater $\mathrm{CO}$ reprocessing have a lower $\mathrm{C} / \mathrm{H}$ ratio in the gas.

After 1 Myr $22 \%$ of models have a $\mathrm{C} / \mathrm{H}$ ratio less than half that predicted by Öberg et al. (2011), increasing to $41 \%$ after $3 \mathrm{Myr}$ and $49 \%$ at both 5 and $6 \mathrm{Myr}$. This suggests planets formed in young, a few megayear old, disks are unlikely to have initial carbon abundances modified by disk chemistry unless there is a strong ionization source. Looking at the low $\mathrm{CO}$ gas abundance in the inner disk of TW Hya, chemistry alone is unlikely to be the sole cause, given the relatively large disk mass $\left(0.05 M_{\odot}\right)$ and low cosmic-ray ionization rate (Cleeves et al. 2015; Schwarz et al. 2016). Our models consider chemistry for a static disk model, without dynamics or an evolving dust population. Such mechanisms must also be at work in order to explain the low observed abundances. As it stands, without the presence of cosmic rays, disk chemistry is unlikely to have a substantial impact on the total gas phase carbon available to forming planets near $12 \mathrm{au}$.

\subsection{2. $\mathrm{O} / \mathrm{H}$}

In this section we analyze the total gas phase $\mathrm{O} / \mathrm{H}$ ratio in the midplane at $12 \mathrm{au}$. To calculate the $\mathrm{O} / \mathrm{H}$ based only on snowline locations we assume $34 \%$ of the total oxygen is in $\mathrm{CO}, 14 \%$ is in $\mathrm{CO}_{2}, 20 \%$ is in $\mathrm{H}_{2} \mathrm{O}$, and the remaining $32 \%$ is in refractory silicates. Between the $\mathrm{CO}$ and $\mathrm{CO}_{2}$ snowlines the predicted $\mathrm{O} / \mathrm{H}$ ratio relative to stellar is 0.34 , increasing to 0.48 inside the $\mathrm{CO}_{2}$ snowline and 0.68 inside the $\mathrm{H}_{2} \mathrm{O}$ snowline.
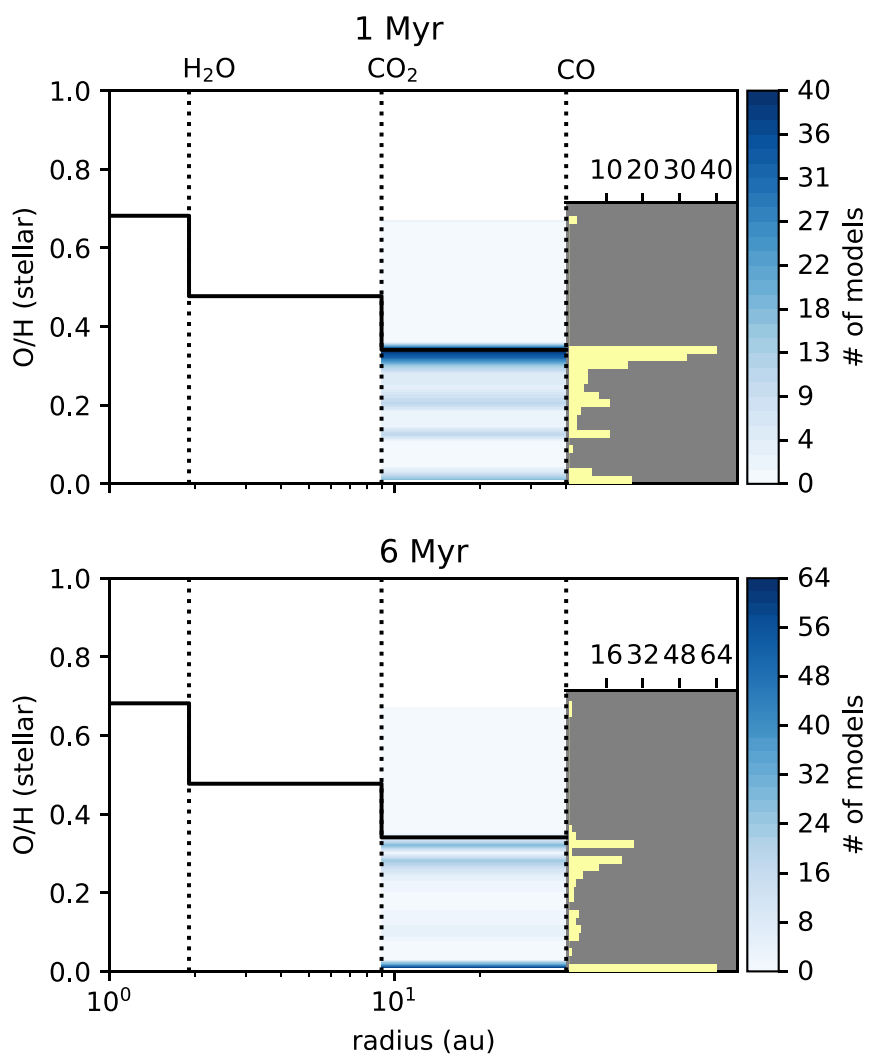

Figure 6. $\mathrm{O} / \mathrm{H}$ ratio in the gas based on the location of major volatile snowlines (line) and the distribution of $\mathrm{O} / \mathrm{H}$ values inside the $\mathrm{CO}$ snowline for our models (shading and histogram) after $1 \mathrm{Myr}$ (top) and $6 \mathrm{Myr}$ (bottom). Radial changes in the $\mathrm{O} / \mathrm{H}$ value are based on the oxygen partitioning assumptions of Öberg et al. (2011).

Comparing this predicted $\mathrm{O} / \mathrm{H}$ profile to the $\mathrm{O} / \mathrm{H}$ gas abundances in our models inside the midplane $\mathrm{CO}$ snowline, the breakdown of models with different $\mathrm{O} / \mathrm{H}$ ratios follows that seen for $\mathrm{C} / \mathrm{H}$ (Figure 6). This is unsurprising since $\mathrm{CO}$ is the dominant gas phase reservoir for both carbon and oxygen. On the whole, the spread of $\mathrm{O} / \mathrm{H}$ values in our models is smaller than for $\mathrm{C} / \mathrm{H}$ since most of the oxygen is in $\mathrm{H}_{2} \mathrm{O}$ ice. There are a handful of models with extremely low $\mathrm{O} / \mathrm{H}$ ratios, significantly lower than the corresponding $\mathrm{C} / \mathrm{H}$, in which there are large reservoirs of $\mathrm{CH}_{3} \mathrm{OH}$ ice in addition to $\mathrm{CO}_{2}$ ice and $\mathrm{H}_{2} \mathrm{O}$ ice. This leads to an excess of carbon with respect to oxygen in the gas, the consequences of which are discussed in the next section.

\subsection{3. $\mathrm{C} / \mathrm{O}$}

Figure 7 shows the midplane $\mathrm{C} / \mathrm{O}$ ratio in the gas at $12 \mathrm{au}$ after $6 \mathrm{Myr}$. Nearly every model has $\mathrm{C} / \mathrm{O} \sim 1$. Only one model, the warm, high cosmic-ray rate model with a disk mass of $0.03 M_{\odot}$ and $99 \%$ of dust in large grains, has a $\mathrm{C} / \mathrm{O}$ ratio below solar. Thus, any planet formed between the $\mathrm{CO}$ and $\mathrm{CO}_{2}$ snowlines observed to have a substellar atmospheric $\mathrm{C} / \mathrm{O}$ would need to obtain at least half of its metals from solids. As this seems unlikely, the atmospheres of such planets should have super-stellar $\mathrm{C} / \mathrm{O}$.

While most models have a midplane $\mathrm{C} / \mathrm{O}$ ratio near unity, the high cosmic-ray rate models with extreme $\mathrm{CO}$ reprocessing have extremely large $\mathrm{C} / \mathrm{O}$ ratios of order $10^{8}$ (Figure 8 ). In 


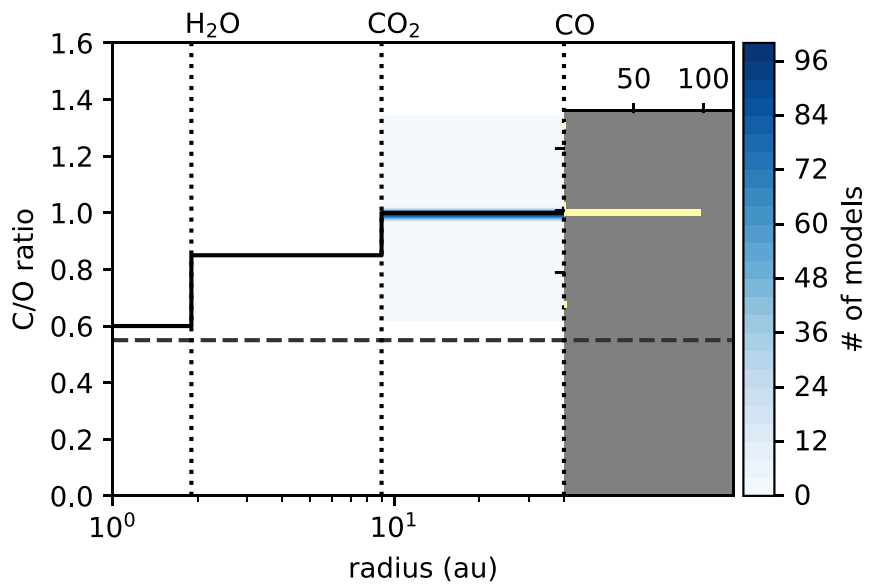

Figure 7. $\mathrm{C} / \mathrm{O}$ ratio in the gas based on the location of major volatile snowlines (line) and the distribution of $\mathrm{C} / \mathrm{O}$ values inside the $\mathrm{CO}$ snowline for our models (shading and histogram) after 6 Myr. Models with extremely large $\mathrm{C} / \mathrm{O}$ are not shown. The gray dashed line indicates $\mathrm{C} / \mathrm{O}$ for the Sun.

these models both the gas phase carbon and oxygen are substantially lower. While most of the carbon is in $\mathrm{CH}_{3} \mathrm{OH}$ ice and $\mathrm{CO}_{2}$ ice, the most abundant gas phase carbon species is $\mathrm{CH}_{4}$ with abundances up to $10^{-7}$. In these models $\mathrm{C}_{2} \mathrm{H}$ is the second most abundant gas phase carbon species, though abundances only reach $10^{-11}$.

Within our time dependent chemical model, the series of chemical reactions leading to $\mathrm{CH}_{4}$ begin when $\mathrm{He}^{+}$reacts with $\mathrm{CO}$ to create $\mathrm{C}^{+}$, which recombines with an electron. The resulting neutral carbon atom reacts with $\mathrm{H}_{2}$ to form $\mathrm{CH}_{2}$, which then freezes out. Successive hydrogenation on the grain surface forms $\mathrm{CH}_{4}$ ice. While the majority of the $\mathrm{CH}_{4}$ molecules remain on the grain surface, some do desorb back into the gas. This population of gas phase hydrocarbons is built up over time, and is not seen at earlier times when the total gas phase $\mathrm{O} / \mathrm{H}$ is high. In these models the gas phase abundances of both carbon and oxygen have been reduced, with the oxygen more depleted than the carbon. Similar reductions in the higher, UV-dominated regions of the disk likewise are needed to match the rings of $\mathrm{C}_{2} \mathrm{H}$ emission observed in TW Hya and DM Tau (Bergin et al. 2016).

The presence of gas phase hydrocarbons at a low abundance results in an extreme enhancement of the gas $\mathrm{C} / \mathrm{O}$ ratio. Sublimation of unprocessed $\mathrm{CO}$ ice from the outer disk as grains drift inward could easily dilute this effect. However, as can be seen in Figures 3 and 4, much of the carbon in the outer disk is chemically reprocessed into less volatile ices, which will not sublimate near the $\mathrm{CO}$ snowline. Additional work is needed in order to understand how the combined effects of dust drift and chemistry change the gas phase $\mathrm{C} / \mathrm{O}$ ratio in the inner disk.

\subsection{Comparison to Comets}

Comets, as the least modified bodies, provide the best record of the composition of volatile ices in the solar nebula. Surveys of primary volatiles in Oort cloud comets reveal high abundances of $\mathrm{CO}_{2}, \mathrm{CO}$, and $\mathrm{CH}_{3} \mathrm{OH}$ relative to water (Mumma \& Charnley 2011). Oort cloud comets formed much closer in, 5-15 au, but were scattered out of their formation region by an early disruption event, e.g., as predicted by the Nice Model (Gomes et al. 2005).

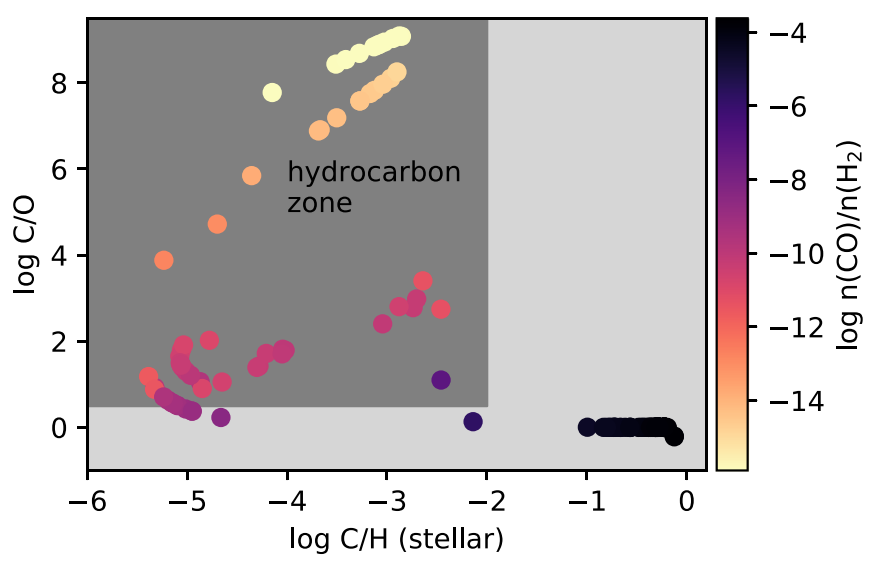

Figure 8. Gas phase $\mathrm{C} / \mathrm{O}$ vs. gas phase $\mathrm{C} / \mathrm{H}$ relative to stellar for the midplane at 12 au after 6 Myr. When cosmic rays are present some of the carbon in $\mathrm{CO}$ is converted into gas phase hydrocarbons, resulting in a large $\mathrm{C} / \mathrm{O}$.

Figure 9 compares the midplane abundances of $\mathrm{CO}_{2}, \mathrm{CO}$, and $\mathrm{CH}_{3} \mathrm{OH}$ ices in our models to the range of values observed in comets. We consider our models with a total disk mass of either $0.1 M_{\odot}$ or $0.03 M_{\odot}$, as the surface density at 12 au in these models bracket the value for the minimum mass solar nebula (Weidenschilling 1977). We do not include the models for which the initial $\mathrm{H}_{2} \mathrm{O}$ abundance was removed, but do include all models with our fiducial initial abundances. Our models already begin with a $\mathrm{CO}_{2}$ ice abundance relative to water greater than that seen in comets, which only increases. In contrast, our models do not start with any $\mathrm{CH}_{3} \mathrm{OH}$ or $\mathrm{CO}$ ice, but the majority of models are able to match the abundance of these ices relative to $\mathrm{H}_{2} \mathrm{O}$ after $1 \mathrm{Myr}$ of chemical evolution. After $6 \mathrm{Myr}$ most models contain more $\mathrm{CH}_{3} \mathrm{OH}$ ice than seen in comets while still matching for $\mathrm{CO}$ ice.

Since $12 \mathrm{au}$ is well outside the midplane $\mathrm{H}_{2} \mathrm{O}$ snowline, the midplane $\mathrm{H}_{2} \mathrm{O}$ ice abundance evolves very little with time. Therefore, we can easily explore the affect of a different initial $\mathrm{H}_{2} \mathrm{O}$ abundance. Increasing the $\mathrm{H}_{2} \mathrm{O}$ ice abundance by a factor of 1.5 brings $\mathrm{CO}_{2}$ ice abundances within the range observed in comets while also keeping $\mathrm{CO}$ and $\mathrm{CH}_{3} \mathrm{OH}$ ices within the cometary range for the majority of the models. Alternatively, starting with less carbon in $\mathrm{CO}_{2}$ ice would lead to $\mathrm{CO}_{2}$ ice abundances within the range observed in comets after some chemical evolution. However, the $\mathrm{CO}$ ice and $\mathrm{CH}_{3} \mathrm{OH}$ ice abundances in such models cannot be predicted without running the chemistry. In summary, the ice abundances in our models are strongly sensitive to the initial conditions and do not provide stringent constraints on the origins of cometary ices. However, the effects seen in our models do demonstrate that the ice abundances in disks can reproduce those observed in comets for a range of physical conditions.

\section{Summary}

We have analyzed the chemical abundances in the midplane at $12 \mathrm{au}$ for 198 unique, physically static, disk models, considering a range of disk mass, large grain fraction, X-ray luminosity, cosmic-ray ionization, temperature, and initial $\mathrm{H}_{2} \mathrm{O}$ abundance with the goal of exploring the range of physical conditions under which carbon can be removed from $\mathrm{CO}$ via chemical reprocessing. We find that: 

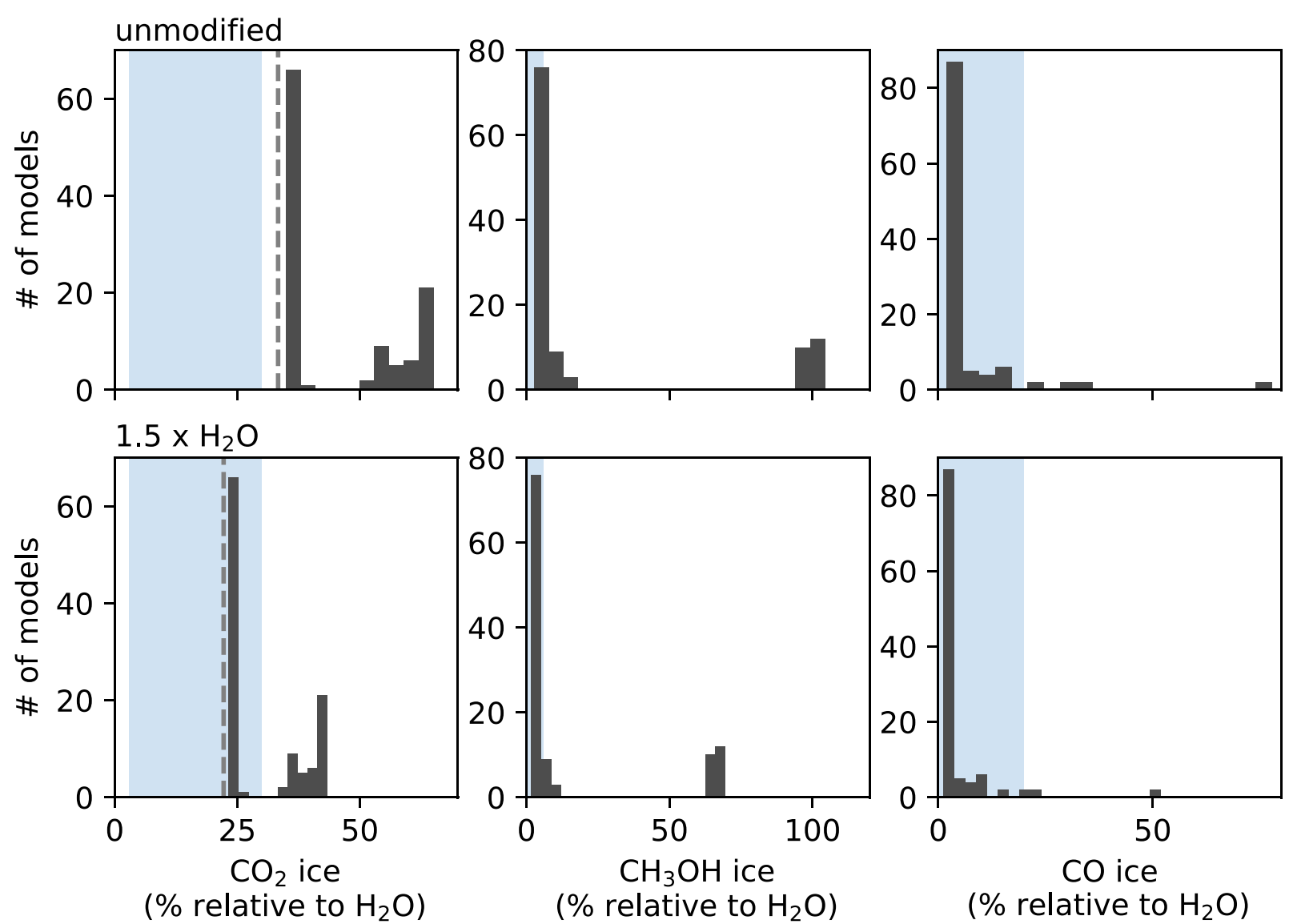

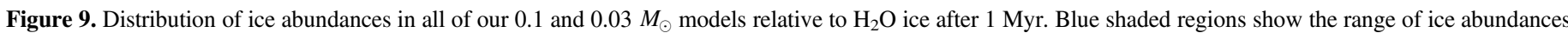
observed in comets and gray dashed lines indicate the initial $\mathrm{CO}_{2}$ abundance. Cometary values are taken from Mumma \& Charnley (2011).

1. Under most conditions an ISM level cosmic-ray ionization rate is needed to reprocess $\mathrm{CO}$ in the midplane, converting it to $\mathrm{CH}_{3} \mathrm{OH}$ ice.

2. In highly dust settled, low surface density disks the presence of UV photons can result in $\mathrm{CO}$ being reprocessed into $\mathrm{CO}_{2}$ ice. Thus disk gas evolution can lead to substantial chemical evolution in the main elemental pools of carbon and oxygen.

3. While most models have gas phase $\mathrm{C} / \mathrm{H}$ and $\mathrm{O} / \mathrm{H}$ abundances close to what is expected based on snowline locations, models with more $\mathrm{CO}$ reprocessing have lower $\mathrm{C} / \mathrm{H}$ and $\mathrm{O} / \mathrm{H}$, with the most depleted models having extremely low $\mathrm{O} / \mathrm{H}$ since the formation of $\mathrm{CO}_{2}$ ice preferentially removes oxygen.

4. The gas phase $\mathrm{C} / \mathrm{O}$ in most models is near unity, with only one model having a subsolar $\mathrm{C} / \mathrm{O}$. Several models have highly elevated $\mathrm{C} / \mathrm{O}$ values. These are models highly depleted in $\mathrm{CO}$ due to the presence of a strong source of ionization, but with a significant reservoir of gas phase hydrocarbons.

5. Our models overproduce $\mathrm{CO}_{2}$ ice relative to $\mathrm{H}_{2} \mathrm{O}$ as compared to cometary abundances, but fall within the range observed for $\mathrm{CH}_{3} \mathrm{OH}$ and $\mathrm{CO}$ ice. Modifying the initial ice abundances allows us to match cometary abundances for all three species in a subset of models.

We conclude that a strong source of ionization or photolysis is needed to chemically reprocess $\mathrm{CO}$ in the disk midplane via in situ processes. While cosmic-ray ionization is unlikely to contribute to the low midplane $\mathrm{CO}$ abundances in TW Hya given its low ionization rate, a more complete understanding of the ionization structure in protoplanetary disks as a population is needed in order the determine the contribution of chemistry to the removal of volatiles from the gas. Since it is extremely difficult for chemical processes to lower the gas phase $\mathrm{C} / \mathrm{O}$ ratio between the $\mathrm{CO}$ and $\mathrm{CO}_{2}$ snowlines, any planets accreting their atmospheres in this region should have super-stellar $\mathrm{C} / \mathrm{O}$ in the absence of other processes.

This work was supported by funding from NSF grant AST1514670 and NASA NNX16AB48G. K.S. and K.Z. acknowledge the support of NASA through Hubble Fellowship Program grants HST-HF2-51419.001 and HST-HF2-51401.001, awarded by the Space Telescope Science Institute, which is operated by the Association of Universities for Research in Astronomy, Inc., for NASA, under contract NAS5-26555.

Software: GNU Parallel (Tange 2011), IDL, matplotlib (Hunter 2007), numpy (van der Walt et al. 2011), pandas (McKinney et al. 2010), scipy (Jones et al. 2001), TORUS (Harries 2000).

\section{Appendix A Truncated Disk}

To test the sensitivity of the midplane chemistry to our choice of outer radius we generated a disk model with the same surface density as our $0.003 M_{\odot}$ and $0.1 M_{\odot}$ disks with $50 \%$ in large grains, but with the outer radius truncated to $30 \mathrm{au}$. We 
low density hiCR

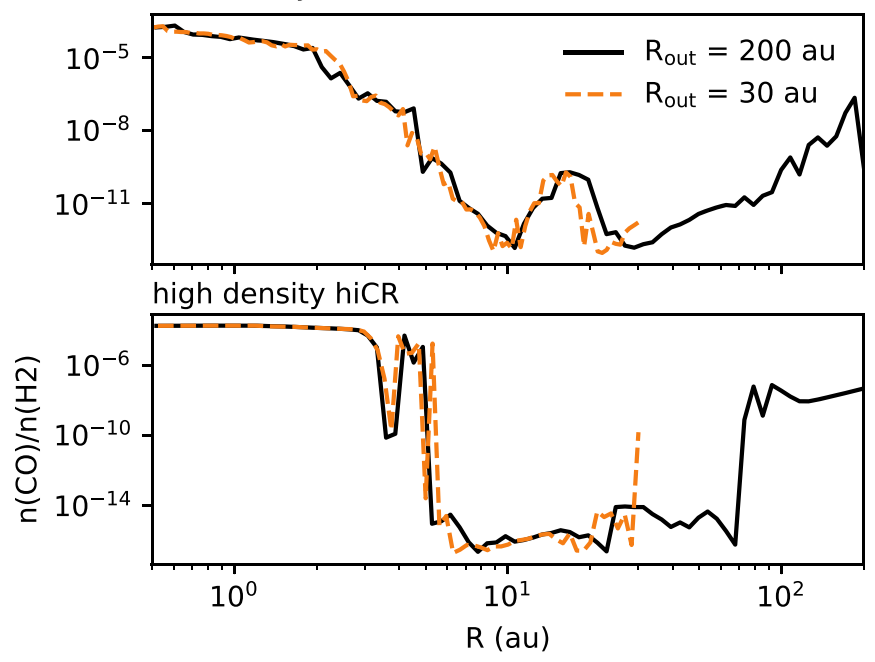

Figure 10. Comparison of midplane $\mathrm{CO}$ gas abundance after $6 \mathrm{Myr}$ of chemical revolution in disks with an outer radius of 200 and 30 au. Top: disks with a surface density equivalent to the surface density in our $0.003 M_{\odot}$ models with an outer radius of $200 \mathrm{au}, 50 \%$ large grains and a high cosmic-ray ionization rate. Bottom: disks with a surface density equivalent to the surface density in our $0.1 M_{\odot}$ models with an outer radius of 200 au, $50 \%$ large grains, and a high cosmic-ray ionization rate. then reran the full radiative transfer and chemistry for a cosmicray ionization rate of $2 \times 10^{-17} \mathrm{~s}^{-1}$ (our high cosmic-ray rate case) and an X-ray luminosity of $10^{30} \mathrm{erg} \mathrm{s}^{-1}$ (our fiducial $\mathrm{X}$-ray luminosity). Figure 10 compares the midplane $\mathrm{CO}$ gas abundances in our truncated disk to the equivalent 200 au disk. There is very little variation in the $\mathrm{CO}$ abundance as a function of disk size. In part, this is because the only source of radiation in our models is the central star. As such radiation does not need to pass through, and be attenuated by, the outer disk in order to reach the inner disk. Additionally, the chemistry at each radius is calculated independently such that the abundances at one radius do not affect abundances at a smaller radius via, e.g., self-shielding. Finally, we note that while our models are physically static, radial drift in an evolving disk could result in different chemical abundances between large and small disks.

\section{Appendix B \\ Abundance Table}

Table 3 lists the five most abundant carbon bearing species in the midplane at 12 au for each model. 
Table 3

Top Five Most Abundant Carbon Bearing Species in the Midplane at 12 au for Each Model after $1 \mathrm{Myr}$

\begin{tabular}{|c|c|c|c|c|c|c|c|c|c|c|c|c|}
\hline Model & 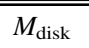 & $\bar{f} f_{l}$ & Species & PAbundance & Species & Abundance & Species & Abundance & Species & ב Abundance & Species & Abundance \\
\hline fiducial & 0.003 & 0.0 & $\mathrm{CO}$ & $1.93 \mathrm{e}-04$ & $\mathrm{CO} 2$ (gr) & $4.24 \mathrm{e}-05$ & $\mathrm{HCN}(\mathrm{gr})$ & $7.76 \mathrm{e}-07$ & $\mathrm{HNC}(\mathrm{gr})$ & $7.34 \mathrm{e}-07$ & $\mathrm{CH} 3 \mathrm{OH}$ (gr) & $5.27 \mathrm{e}-07$ \\
\hline fiducial & 0.003 & 0.1 & $\mathrm{CO}$ & $1.94 \mathrm{e}-04$ & $\mathrm{CO} 2(\mathrm{gr})$ & $4.21 \mathrm{e}-05$ & $\mathrm{HCN}(\mathrm{gr})$ & $7.63 \mathrm{e}-07$ & $\mathrm{HNC}(\mathrm{gr})$ & $7.28 \mathrm{e}-07$ & $\mathrm{CH} 3 \mathrm{OH}$ (gr) & $4.97 \mathrm{e}-07$ \\
\hline fiducial & 0.003 & 0.2 & $\mathrm{CO}$ & $1.94 \mathrm{e}-04$ & $\mathrm{CO} 2(\mathrm{gr})$ & $4.22 \mathrm{e}-05$ & $\mathrm{HCN}(\mathrm{gr})$ & $7.46 \mathrm{e}-07$ & $\mathrm{HNC}(\mathrm{gr})$ & $7.15 \mathrm{e}-07$ & $\mathrm{CH} 3 \mathrm{OH}(\mathrm{gr})$ & $5.57 \mathrm{e}-07$ \\
\hline fiducial & 0.003 & 0.3 & $\mathrm{CO}$ & $1.92 \mathrm{e}-04$ & $\mathrm{CO} 2(\mathrm{gr})$ & $4.30 \mathrm{e}-05$ & $\mathrm{CH} 3 \mathrm{OH}(\mathrm{gr})$ & $8.44 \mathrm{e}-07$ & $\mathrm{HCN}(\mathrm{gr})$ & $6.93 e-07$ & $\mathrm{HNC}(\mathrm{gr})$ & $6.70 \mathrm{e}-07$ \\
\hline fiducial & 0.003 & 0.4 & $\mathrm{CO}$ & $1.88 \mathrm{e}-04$ & $\mathrm{CO} 2(\mathrm{gr})$ & $4.40 \mathrm{e}-05$ & $\mathrm{CH} 3 \mathrm{OH}(\mathrm{gr})$ & $1.25 \mathrm{e}-06$ & $\mathrm{HCN}(\mathrm{gr})$ & $6.10 \mathrm{e}-07$ & $\mathrm{HNC}(\mathrm{gr})$ & $5.97 \mathrm{e}-07$ \\
\hline fiducial & 0.003 & 0.5 & $\mathrm{CO}$ & $1.88 \mathrm{e}-04$ & $\mathrm{CO} 2(\mathrm{gr})$ & $4.42 \mathrm{e}-05$ & $\mathrm{CH} 3 \mathrm{OH}(\mathrm{gr})$ & $1.21 \mathrm{e}-06$ & $\mathrm{HCN}(\mathrm{gr})$ & $7.14 \mathrm{e}-07$ & $\mathrm{HNC}(\mathrm{gr})$ & $6.70 \mathrm{e}-07$ \\
\hline fiducial & 0.003 & 0.6 & $\mathrm{CO}$ & $1.87 \mathrm{e}-04$ & $\mathrm{CO} 2$ (gr) & $4.45 \mathrm{e}-05$ & $\mathrm{CH} 3 \mathrm{OH}(\mathrm{gr})$ & $1.48 \mathrm{e}-06$ & C3H4(gr) & $5.85 \mathrm{e}-07$ & $\mathrm{HCN}(\mathrm{gr})$ & $5.43 \mathrm{e}-07$ \\
\hline fiducial & 0.003 & 0.7 & $\mathrm{CO}$ & $1.82 \mathrm{e}-04$ & $\mathrm{CO} 2(\mathrm{gr})$ & $4.60 \mathrm{e}-05$ & $\mathrm{CH} 3 \mathrm{OH}(\mathrm{gr})$ & $2.30 \mathrm{e}-06$ & $\mathrm{C} 3 \mathrm{H} 4$ (gr) & $8.03 e-07$ & $\mathrm{HC} 3 \mathrm{~N}(\mathrm{gr})$ & $5.58 \mathrm{e}-07$ \\
\hline fiducial & 0.003 & 0.8 & $\mathrm{CO}$ & $1.79 \mathrm{e}-04$ & $\mathrm{CO} 2(\mathrm{gr})$ & $4.67 e-05$ & $\mathrm{CH} 3 \mathrm{OH}(\mathrm{gr})$ & $2.76 \mathrm{e}-06$ & $\mathrm{C} 3 \mathrm{H} 4$ (gr) & $9.25 \mathrm{e}-07$ & $\mathrm{HC} 3 \mathrm{~N}(\mathrm{gr})$ & $7.53 \mathrm{e}-07$ \\
\hline fiducial & 0.003 & 0.9 & $\mathrm{CO}$ & $1.74 \mathrm{e}-04$ & $\mathrm{CO} 2(\mathrm{gr})$ & $4.66 \mathrm{e}-05$ & $\mathrm{CH} 3 \mathrm{OH}(\mathrm{gr})$ & $3.91 \mathrm{e}-06$ & $\mathrm{HC} 3 \mathrm{~N}(\mathrm{gr})$ & $1.36 \mathrm{e}-06$ & $\mathrm{C} 3 \mathrm{H} 4$ (gr) & $1.18 \mathrm{e}-06$ \\
\hline
\end{tabular}

Note. Abundances are relative to $\mathrm{H}_{2}$.

(This table is available in its entirety in machine-readable form.) 


\section{ORCID iDs}

Kamber R. Schwarz (1) https://orcid.org/0000-00026429-9457

Edwin A. Bergin (10) https://orcid.org/0000-0003-4179-6394

L. Ilsedore Cleeves (i) https://orcid.org/0000-0003-2076-8001

Ke Zhang (i) https://orcid.org/0000-0002-0661-7517

Karin I. Öberg (i) https://orcid.org/0000-0001-8798-1347

Geoffrey A. Blake (i) https://orcid.org/0000-0003-0787-1610

Dana E. Anderson (1) https://orcid.org/0000-0002-8310-0554

\section{References}

Aikawa, Y., \& Herbst, E. 1999, A\&A, 351, 233

Anderl, S., Maret, S., Cabrit, S., et al. 2016, A\&A, 591, A3

Ansdell, M., Williams, J. P., van der Marel, N., et al. 2016, ApJ, 828, 46

Bai, X.-N. 2016, ApJ, 821, 80

Bergin, E. A., Cleeves, L. I., Gorti, U., et al. 2013, Natur, 493, 644

Bergin, E. A., Du, F., Cleeves, L. I., et al. 2016, ApJ, 831, 101

Bethell, T. J., \& Bergin, E. A. 2011a, ApJ, 740, 7

Bethell, T. J., \& Bergin, E. A. 2011b, ApJ, 739, 78

Booth, R. A., Clarke, C. J., Madhusudhan, N., \& Ilee, J. D. 2017, MNRAS, 469, 3994

Bosman, A. D., Walsh, C., \& van Dishoeck, E. F. 2018, A\&A, 618, A182

Chabrier, G., Johansen, A., Janson, M., \& Rafikov, R. 2014, in Protostars and Planets VI, ed. H. Beuther et al. (Tucson, AZ: Univ. Arizona Press), 619

Ciesla, F. J., \& Cuzzi, J. N. 2006, Icar, 181, 178

Cleeves, L. I., Bergin, E. A., \& Adams, F. C. 2014a, ApJ, 794, 123

Cleeves, L. I., Bergin, E. A., Alexander, C. M. O., et al. 2014b, Sci, 345, 1590

Cleeves, L. I., Bergin, E. A., Qi, C., Adams, F. C., \& Öberg, K. I. 2015, ApJ, 799, 204

Cridland, A. J., Pudritz, R. E., Birnstiel, T., Cleeves, L. I., \& Bergin, E. A. 2017, MNRAS, 469, 3910

Dodson-Robinson, S. E., Evans, N. J., II, Ramos, A., Yu, M., \& Willacy, K. 2018, ApJL, 868, L37

Du, F., Bergin, E. A., Hogerheijde, M., et al. 2017, ApJ, 842, 98

Eistrup, C., Walsh, C., \& van Dishoeck, E. F. 2016, A\&A, 595, A83

Eistrup, C., Walsh, C., \& van Dishoeck, E. F. 2018, A\&A, 613, A14

Espinoza, N., Fortney, J. J., Miguel, Y., Thorngren, D., \& Murray-Clay, R. 2017, ApJL, 838, L9

Estrada, P. R., Cuzzi, J. N., \& Morgan, D. A. 2016, ApJ, 818, 200

Favre, C., Cleeves, L. I., Bergin, E. A., Qi, C., \& Blake, G. A. 2013, ApJL, 776, L38
Fogel, J. K. J., Bethell, T. J., Bergin, E. A., Calvet, N., \& Semenov, D. 2011, ApJ, 726, 29

Gomes, R., Levison, H. F., Tsiganis, K., \& Morbidelli, A. 2005, Natur, 435, 466

Harries, T. J. 2000, MNRAS, 315, 722

Hunter, J. D. 2007, CSE, 9, 90

Jones, E., Oliphant, T., Peterson, P., et al. 2001, SciPy: Open Source Scientific Tools for Python, http://www.scipy.org/

Kama, M., Bruderer, S., Carney, M., et al. 2016, A\&A, 588, A108

Kreidberg, L., Line, M. R., Bean, J. L., et al. 2015, ApJ, 814, 66

Krijt, S., \& Ciesla, F. J. 2016, ApJ, 822, 111

Krijt, S., Schwarz, K. R., Bergin, E. A., \& Ciesla, F. J. 2018, ApJ, 864, 78

Lavie, B., Mendonça, J. M., Mordasini, C., et al. 2017, AJ, 154, 91

Long, F., Herczeg, G. J., Pascucci, I., et al. 2017, ApJ, 844, 99

Mathis, J. S., Rumpl, W., \& Nordsieck, K. H. 1977, ApJ, 217, 425

McClure, M. K., Bergin, E. A., Cleeves, L. I., et al. 2016, ApJ, 831, 167

McElroy, D., Walsh, C., Markwick, A. J., et al. 2013, A\&A, 550, A36

McKinney, W., Anders, M., Barber, S. K., et al. 2010, Proc. SPIE, 7801, 780106

Meijerink, R., Pontoppidan, K. M., Blake, G. A., Poelman, D. R., \& Dullemond, C. P. 2009, ApJ, 704, 1471

Miotello, A., Bruderer, S., \& van Dishoeck, E. F. 2014, A\&A, 572, A96

Molyarova, T., Akimkin, V., Semenov, D., et al. 2017, ApJ, 849, 130

Mumma, M. J., \& Charnley, S. B. 2011, ARA\&A, 49, 471

Öberg, K. I., \& Bergin, E. A. 2016, ApJL, 831, L19

Öberg, K. I., Murray-Clay, R., \& Bergin, E. A. 2011, ApJL, 743, L16

Padovani, M., Marcowith, A., Hennebelle, P., \& Ferrière, K. 2016, A\&A, 590, A8

Piso, A.-M. A., Öberg, K. I., Birnstiel, T., \& Murray-Clay, R. A. 2015, ApJ, 815,109

Reboussin, L., Wakelam, V., Guilloteau, S., Hersant, F., \& Dutrey, A. 2015, A\&A, 579, A82

Schwarz, K. R., Bergin, E. A., Cleeves, L. I., et al. 2016, ApJ, 823, 91

Schwarz, K. R., Bergin, E. A., Cleeves, L. I., et al. 2018, ApJ, 856, 85

Smith, I. W. M., Herbst, E., \& Chang, Q. 2004, MNRAS, 350, 323

Tange, O. 2011, login: The USENIX Magazine, 36, 42

Tychoniec, Ł., Tobin, J. J., Karska, A., et al. 2018, ApJS, 238, 19

van der Walt, S., Colbert, S. C., \& Varoquaux, G. 2011, CSE, 13, 22

van't Hoff, M. L. R., Tobin, J. J., Harsono, D., \& van Dishoeck, E. F. 2018 , A\&A, 615, A83

Weidenschilling, S. J. 1977, Ap\&SS, 51, 153

Weingartner, J. C., \& Draine, B. T. 2001, ApJS, 134, 263

Williams, J. P., \& Best, W. M. J. 2014, ApJ, 788, 59

Xu, R., Bai, X.-N., \& Öberg, K. 2017, ApJ, 835, 162

Yu, M., Willacy, K., Dodson-Robinson, S. E., Turner, N. J., \& Evans, N. J., II 2016, ApJ, 822, 53

Zhang, K., Bergin, E. A., Blake, G. A., Cleeves, L. I., \& Schwarz, K. R. 2017, NatAs, 1, 0130 